Published in final edited form as:

Nat Clin Pract Cardiovasc Med. 2005 November ; 2(11): 576-584. doi:10.1038/ncpcardio0348.

\title{
Drug Insight:
}

\section{statins and stroke}

\section{Ralph L Sacco and James K Liao*}

JK Liao is Director of Vascular Medicine Research at Brigham \& Women's Hospital, Cambridge, MA, USA, and Associate Professor of Medicine at Harvard Medical School, MA, USA. RL Sacco is Director of the Stroke and Critical Care Division, and Professor of Neurology and Epidemiology at the Neurological Institute of Columbia University College of Physicians and Surgeons, the Mailman School of Public Health, New York, and the Sergievsky Center, New York, NY, USA

\section{SUMMARY}

Stroke is the third leading cause of death in the US and a common cause of long-term disability worldwide. Ischemic strokes, which are often atherothrombotic, account for more than $80 \%$ of all strokes. Current stroke prevention focuses on optimizing the treatment of modifiable risk factors, such as hypertension, diabetes and dyslipidemia. The epidemiologic association between serum cholesterol levels and adjusted stroke rates is not as strong as the link between serum cholesterol levels and coronary heart disease. Clinical trials of 3-hydroxy-3-methylglutaryl-coenzyme A reductase inhibitors (statins), which are potent inhibitors of cholesterol synthesis, have demonstrated, however, a marked reduction in stroke risk in hypercholesterolemic and atherosclerotic individuals, with benefits extending to normocholesterolemic individuals. These findings suggest that statins might have additional effects in stroke protection beyond cholesterol reduction. Because statins inhibit the synthesis of isoprenoid intermediates in the cholesterol biosynthetic pathway, which are important lipid attachments for intracellular signaling molecules, they might have direct noncholesterol-dependent effects on inflammatory and endothelial cells. Here we discuss data from clinical trials assessing the effects of statins on stroke risk, as well as outline the mechanisms evidence-based recommendations for stroke prevention, based on achieved underlying the cholesterol-independent effects of statins and provide serum cholesterol levels in patients at risk of stroke.

\section{Keywords}

cholesterol; endothelium; nitric oxide; statins; stroke

\section{INTRODUCTION}

Stroke has a major impact on the public health of every nation. In the US, stroke is the third leading cause of death, accounting for 160,000 deaths per year, and is the most common cause

\footnotetext{
(C)2005 Nature Publishing Group

*Correspondence Vascular Medicine Research, Brigham \& Women's Hospital, 65 Landsdowne Street, Room 275, Cambridge, MA 02139, USA, jliao@ rics.bwh.harvard.edu

Competing interests

The authors declared competing interests; go to the article online for details.

REVIEW CRITERIA

All articles were identified by searching the MEDLINE and PubMed databases. The search keywords used were "statins" and "stroke". All articles were full-text, English-language clinical trials and meta-analyses based on human studies.
} 
of long-term disability. Each year, 700,000 Americans suffer a new or recurrent stroke. Nearly two-thirds of ischemic strokes are atherothrombotic, resulting from atherosclerosis of large arteries, or vasculopathy of small arteries. ${ }^{1}$ Cerebral ischemia results from reduced blood flow in a narrowed or occluded artery, or an embolism from a complex atherosclerotic plaque. The cause of the narrowing is most often atherosclerosis, a process involving endothelial injury, inflammation, lipid deposition, smooth-muscle-cell proliferation, plaque formation, fibrin, platelets and thrombin.

\section{LIPIDS AND RISK FACTORS FOR STROKE}

Abnormal levels of serum lipids, including triglycerides, LDL and HDL cholesterol, are regarded as greater risk factors for coronary artery disease (CAD) than for cerebrovascular disease. Hypercholesterolemia might promote atherosclerosis, because elevated concentrations of oxidized or modified LDL cholesterol contribute to endothelial dysfunction.

2,3 The association between hypercholesterolemia and stroke has become more apparent because of data from prospective cohort studies that show higher risks of ischemic stroke with increasing levels of total cholesterol in both men and women. ${ }^{4-7}$ Increased HDL cholesterol levels have a protective effect against the occurrence of ischemic stroke, ${ }^{8,9}$ but the association between LDL cholesterol and stroke is less clear. Elevated triglyceride levels have also been reported as a risk factor for stroke. ${ }^{7,10}$ Overall, elevated total cholesterol confers an approximately twofold relative increase in stroke risk for men and women. ${ }^{11}$ Moreover, the high prevalence of this condition in the population translates into a larger proportion of strokes potentially attributable to dyslipidemia and, therefore, a greater relative importance in public health. ${ }^{11}$

Thus, current stroke-prevention strategies include management of modifiable risk factors, including hypertension, diabetes and hypercholesterolemia, which affect atherosclerosis and thrombus formation. ${ }^{11-13}$ Evidence from clinical trials indicates that statins lower serum cholesterol levels and reduce the risk of stroke. The primary mechanism of action of statins is the competitive inhibition of 3-hydroxy-3-methylglutaryl-coenzyme A reductase, the enzyme that catalyzes the rate-limiting step in cholesterol biosynthesis. Inhibition of this enzyme results in increased expression of the LDL cholesterol receptor in the liver, leading to increased clearance of LDL cholesterol and its precursors, as well as protection against oxidized LDL cholesterol. In addition to their actions on cholesterol biosynthesis, statins might have other effects, including improvements in endothelial function, reduction of inflammation, stabilization and regression of atherosclerotic plaques, attenuation of thrombogenic responses and increases in circulating endothelial progenitor cells. Indeed, in a study of patients with heart failure that compared the vascular protective effects of a statin and ezetimibe (an inhibitor of intestinal cholesterol absorption), the statin group demonstrated improved endothelial function, enhanced antioxidative properties and increased circulating endothelial progenitor cells, despite similar reductions in LDL cholesterol of $15 \%$ that were seen in both groups. ${ }^{14}$ These findings suggest that there are beneficial effects with statin use beyond lowering LDL cholesterol that could contribute to their effects on stroke protection.

\section{CLINICAL TRIALS OF STATINS IN STROKE PREVENTION}

\section{Statins and subclinical carotid disease}

Statins decrease the risk of various cardiovascular outcomes, particularly stroke (Table 1). Indeed, several clinical trials with statins have shown delayed progression or even regression of subclinical carotid disease assessed by measurement of intima-media thickness. These clinical trials were designed to determine whether control of modest elevations of LDL cholesterol would retard the progression of asymptomatic carotid atherosclerotic plaques. A 
small reduction in carotid artery intima-media thickness has been documented with aggressive lowering of lipid levels in various trials with different statins. ${ }^{15-17}$

\section{Statins and the risk of stroke in patients with coronary disease}

Early clinical trials demonstrated that statin therapy decreased the risk of stroke, particularly among patients with $\mathrm{CAD}$. This reduction was significant in the three major trials that evaluated statin use in patients with existing CAD: the Scandinavian Simvastatin Survival Study (4S), 18 the Long-term Intervention with Pravastatin in Ischemic Disease (LIPID) trial, ${ }^{19}$ and the Cholesterol And Recurrent Events (CARE) trial. ${ }^{20}$ In the 4 S trial, 4,444 patients with angina pectoris or previous myocardial infarction (MI) and total serum cholesterol levels of 5.5-8.0 $\mathrm{mM} / \mathrm{l}(212.4-308.9 \mathrm{mg} / \mathrm{dl})$ on a lipid-lowering diet were randomized to double-blind treatment with 20-40 mg simvastatin or placebo. Post hoc analysis showed a significant reduction in fatal and nonfatal stroke among patients with hyper cholesterolemia who were treated with simvastatin in comparison with those receiving placebo, as well as a reduction in total mortality and cardiac events in this patient group. The risk of stroke was reduced by $30 \%$ in patients receiving simvastatin who had suffered a single MI and had elevated cholesterol levels.

The risk of stroke was also significantly reduced following treatment with pravastatin in the CARE and LIPID trials, in which stroke was prespecified as a secondary endpoint. In the CARE trial, ${ }^{20}$ the effect of lowering cholesterol with $40 \mathrm{mg}$ pravastatin daily on the incidence of vascular events was assessed in patients with a total cholesterol level of less than $6.2 \mathrm{mM} / \mathrm{l}$ $(240 \mathrm{mg} / \mathrm{dl})$ and an LDL cholesterol level of $2.98-4.51 \mathrm{mM} / \mathrm{l}(115-174 \mathrm{mg} / \mathrm{dl} ; n=4,159: 3,583$ men and 576 women). The risk of stroke in patients receiving pravastatin was reduced by $31 \%$ (95\% CI 3-52; $P=0.03$ ) in comparison with those receiving placebo. In addition, this study randomized patients without elevated baseline blood cholesterol levels, and the results suggest that all survivors of a first MI should be considered for cholesterol-lowering therapy to reduce the risk of stroke and ischemic heart disease. The LIPID trial ${ }^{19}$ randomized 9,014 men and women aged 31-75 years with pre-existing coronary heart disease (CHD), either acute MI or unstable angina, and total cholesterol levels of 4-7 mM/l (155-270 mg/dl). Patients in the LIPID trial who received pravastatin ( $40 \mathrm{mg}$ daily) achieved reductions of $24 \%$ in CHD mortality, $22 \%$ in total mortality and $19 \%$ in the incidence of stroke $(P=0.048)$ in comparison with patients who received placebo. Moreover, $82 \%$ of participants were taking aspirin; therefore, pravastatin reduces the risk of these events even in patients who are already receiving antiplatelet therapy.

The benefits of statins for stroke and cardiovascular disease prevention were more broadly evaluated in the large-scale, long-term Heart Protection Study (HPS). ${ }^{21,22}$ In this study, 20,536 adults aged 40-80 years with coronary disease, other occlusive arterial disease, diabetes or treated hypertension were randomly allocated to $40 \mathrm{mg}$ simvastatin daily or placebo. All-cause mortality was reduced among patients receiving simvastatin in comparison with those receiving placebo $(1,328$ [12.9\%] deaths versus 1,507 [14.7\%] deaths, $P=0.0003)$, the rate of nonfatal MI or coronary death was reduced (898 [8.7\%] versus 1,212 [11.8\%], $P<0.0001)$. Fewer patients receiving simvastatin had a nonfatal or fatal stroke than patients receiving placebo (444 [4.3\%] versus $585[5.7 \%]$ patients, $P<0.0001)$.

In a subgroup analysis from the HPS of the 3,280 individuals with cerebrovascular disease, there was no apparent reduction in the stroke rate, which challenged the efficacy of statins in the secondary prevention of stroke. In the HPS, ${ }^{22}$ however, there was a $20 \%$ reduction in the rate of any major vascular event among cerebro vascular disease patients receiving statin therapy rather than placebo (406 [24.7\%] versus 488 [29.8\%] events, $P=0.001)$. The risk of stroke was reduced by about $25 \%$ in many other subgroups, including those with coronary disease or diabetes, those who were younger or older than 70 years of age at study entry, and those presenting with various levels of blood pressure or lipids, even when pretreatment LDL 
cholesterol was below $3.0 \mathrm{mM} / \mathrm{l}(116 \mathrm{mg} / \mathrm{dl})$. The reduction in stroke incidence was not significant during the first year, but was significant $(P=0.0004)$ by the end of the second year. The HPS was notable because it showed benefits of statin use in a wide range of high-risk patients, including those without a diagnosis of CHD.

Primary prevention trials also showed protective effects for statins that were more pronounced for CAD endpoints than for stroke. In the West of Scotland Coronary Prevention Study (WOSCOPS), ${ }^{23} 6,595$ men with hyper cholesterolemia (mean cholesterol level $7.05 \mathrm{mM} / 1$ [ 272 $\mathrm{mg} / \mathrm{dl}]$ ) were randomized to pravastatin or placebo to determine the effects of these regimens on the incidence of nonfatal MI and death from CHD. The incidence of these primary endpoints was significantly reduced; however, stroke, which was a secondary endpoint and underpowered for the study, was only reduced by a nonsignificant $11 \%$. In the Prospective Study of Pravastatin in the Elderly at Risk (PROSPER) trial, ${ }^{24}$ the benefits of pravastatin (40 mg daily) or placebo treatment were evaluated in an elderly cohort of 5,804 men and women aged 70-82 years with a history of, or risk factors for, vascular disease. The primary endpoint was a composite of coronary death, nonfatal MI and fatal or nonfatal stroke. Those patients who were randomized to receive pravastatin had a significantly reduced incidence of the primary composite endpoint in comparison with patients receiving placebo (408 events versus 473 events, hazard ratio (HR) $0.85,95 \% \mathrm{CI} 0.74-0.97 ; P=0.014)$. The risk of coronary disease and nonfatal MI was significantly lower in the pravastatin-treated patients than in those receiving placebo (HR 0.81, 95\% CI 0.69-0.94; $P=0.006)$. The risk of stroke was not significantly different between the two treatment groups (HR 1.03, 95\% CI 0.81-1.31; $P=0.8$ ), but the HR for transient ischemic attack was 0.75 (95\% CI 0.55-1.00; $P=0.051)$.

\section{Statins and the risk of stroke in patients with hypertension or diabetes}

Beneficial effects on the primary prevention of stroke have been observed for statin use among patients with hypertension or diabetes. The Anglo-Scandinavian Cardiac Outcomes Trial-Lipid Lowering Arm (ASCOT-LLA) study ${ }^{25}$ evaluated the benefits of cholesterol lowering in the primary prevention of CHD among hypertensive patients who were not classified as dys lipidemic. Of 19,342 hypertensive patients (aged 40-79 years, with at least three other cardiovascular risk factors), 10,305 with nonfasting total cholesterol concentrations of $6.5 \mathrm{mM} /$ $1(251 \mathrm{mg} / \mathrm{dl})$ or less were randomly assigned to receive atorvastatin $10 \mathrm{mg}$ or placebo daily. The primary endpoints were prespecified as nonfatal MI and fatal CHD. The trial was stopped after a median follow-up of 3.3 years. The occurrence of primary events was significantly reduced in the atorvastatin group in comparison with the placebo group (100 versus 154 events, HR $0.64 ; 95 \%$ CI $0.50-0.83 ; P=0.0005$ ). Fatal and nonfatal stroke were also significantly reduced in the atorvastatin group compared with the placebo group ( 89 versus 121 events, HR $0.73 ; 95 \%$ CI $0.56-0.96 ; P=0.024)$.

In the Collaborative Atorvastatin Diabetes Study (CARDS), ${ }^{26} 2,838$ patients from 132 centers in the UK and Ireland, who were aged 40-75 years and had type 2 diabetes but no cardiovascular disease, were randomized to placebo $(n=1,410)$ or $10 \mathrm{mg}$ atorvastatin daily $(n=1,428)$. The trial was stopped early after finding clear efficacy in an interim analysis after a median followup duration of 3.9 years. Patients allocated to receive atorvastatin had a significantly lower rate of cardiovascular events than those who received placebo (37\% relative rate reduction; $95 \%$ CI -52 to $-17 ; P=0.001)$ and a $48 \%$ relative reduction in the stroke rate $(95 \%$ CI -69 to -11$)$.

The effect of pravastatin on all-cause mortality was evaluated in the Antihypertensive and Lipid-Lowering Treatment to Prevent Heart Attack Trial (ALLHAT) ${ }^{27}$ in 10,355 patients over 55 years of age who were hypertensive and moderately hypercholesterolemic with at least one additional risk factor. Pravastatin did not significantly reduce all-cause mortality, or the incidence of coronary events or stroke, when compared with usual care (in this trial, usual care was defined as including other measures to reduce cholesterol but excluding statin therapy). 
In the course of the study, however, nearly one-third of those allocated to usual care were treated with lipid-lowering drugs, which resulted in a smaller differential in total cholesterol reduction between the two groups.

\section{Early effects of statin therapy}

Most long-term trials assessing statin therapies have shown benefits in terms of stroke reduction within the first year of treatment. Among high-risk patients with unstable angina or non-Qwave MI, the benefits of statin therapy were demonstrated within 16 weeks in the Myocardial Ischemia Reduction with Aggressive Cholesterol Lowering (MIRACL) trial. ${ }^{28}$ The primary endpoint - a composite of death, nonfatal MI, resuscitated cardiac arrest or recurrent symptomatic myocardial ischemia - was reduced from $17.4 \%$ in the placebo group to $14.8 \%$ in the atorvastatin group over the 16 weeks of the trial $(P=0.048)$. Stroke was a secondary endpoint, and the incidence of nonfatal stroke was reduced in the statin treatment group (relative risk $0.40,95 \% \mathrm{CI} 0.19-0.88 ; P=0.02$ ), as was the combined incidence of a fatal or nonfatal stroke (relative risk $0.49,95 \%$ CI $0.24-0.98 ; P=0.04$ ).

\section{Results from meta-analyses and other studies}

Meta-analyses have demonstrated an overall reduced incidence of stroke with statin therapy. 29-31 In a systematic review and meta-analysis of all randomized statin trials published before August 2003, which included over 90,000 patients, the relative risk reduction for stroke was $21 \%$ (odds ratio $0.79,95 \%$ CI $0.73-0.85$ ) with no heterogeneity between trials. ${ }^{32}$ For each $10 \%$ reduction in LDL cholesterol (i.e. $1 \mathrm{mM} / \mathrm{l}$ ), there was an estimated reduction in the risk of stroke of $15.6 \%$ (95\% CI $6.7-23.6)$ and a $0.73 \%$ reduction in carotid intima-media thickness per year (95\% CI 0.27-1.19).

The benefits of statins for secondary stroke prevention among patients with normal cholesterol levels and without CAD remain unclear. The ongoing Stroke Prevention by Aggressive Reduction in Cholesterol Levels (SPARCL) trial ${ }^{33}$ is addressing this question. This trial has enrolled 4,700 patients with previous stroke or transient ischemic attack who have been randomized to atorvastatin ( $80 \mathrm{mg}$ daily) or placebo. The primary endpoint for SPARCL is fatal or nonfatal stroke, and the planned follow-up for the trial is 5 years.

\section{STATIN PLEIOTROPY AND POTENTIAL NONCHOLESTEROL-RELATED EFFECTS ON STROKE}

As mentioned previously, an elevated level of LDL cholesterol has not traditionally been considered an important risk factor for stroke; for example, in the Framingham Heart Study or the Multiple Risk Factor Intervention Trial (MRFIT). ${ }^{34,35}$ Indeed, a review of 45 prospective observational cohorts involving 450,000 individuals failed to find an association between adjusted stroke rates and serum cholesterol levels. ${ }^{36}$ Furthermore, cholesterol reduction in numerous nonstatin trials has generally failed to reduce the incidence of fatal strokes significantly. ${ }^{29}$ Meta-analyses of clinical trials ${ }^{30,31}$ and a post hoc analysis of the data from $4 \mathrm{~S}^{18}$ have, however, shown a clear association between statin therapy and a reduced incidence of cerebrovascular events. Furthermore, data from the HPS 21,22 have shown a $27 \%$ reduction in total stroke incidence (including ischemic stroke) in patients treated with simvastatin $40 \mathrm{mg}$ daily compared with those receiving placebo, a finding that was independent of age, sex and baseline cholesterol level. Collectively, these data suggest that statins might reduce stroke incidence by mechanisms beyond cholesterol lowering. Some of these mechanisms include improvements in endothelial function, reduced inflammation, stabilization and regression of atherosclerotic plaques, and attenuation of thrombogenic responses (Box 1). 


\section{Improved endothelial function}

One of the best-characterized benefits of statin therapy is an improvement in endothelial function, which can occur through a cholesterol-dependent or a cholesterol-independent mechanism. In this context, cholesterol apheresis alone can improve endothelial function within hours of lowering LDL cholesterol levels. ${ }^{37}$ The mechanism by which statins improve endothelial function is thought to involve a nitric oxide (NO)-dependent process. Withdrawal of statin therapy leads to decreased endothelial NO production. ${ }^{38} \mathrm{NO}$ regulates the paracrine antiatherosclerotic functions of the endothelium. Reducing NO synthesis with an inhibitor of endothelial nitric oxide synthase (eNOS) promotes the progression of atherosclerosis ${ }^{39}$ and increases stroke size. ${ }^{40}$

By inhibition of mevalonate synthesis, statins prevent the production of biologically active intermediates, such as the isoprenoids, farnesylpyrophosphate and geranylgeranyl pyrophosphate (Figure 1). Farnesylpyrophosphate and geranylgeranylpyrophosphate have important roles in the post-translational modification of proteins, such as the $\gamma$-subunit of heterotrimeric ${ }_{\mathrm{G} \text { Proteins }}$ and members of the ras suPerfamily of GTP-binding proteins, including Ras, Rho, Rap, Ral, Rab and Rac. Members of the Ras superfamily function as molecular switches that cycle between the inactive GDP-bound state and the active GTP-bound state. Statins inhibit the isoprenYlation of Ras and Rho, thereby promoting an accumulation of the inactive forms of these proteins within the cytoplasm of endothelial cells. It is the inhibition of Rho isoprenylation by statins that is largely responsible for the upregulation of eNOS expression within the endothelium. ${ }^{41,42}$ Statins can, however, also directly activate eNOS through additional post-translational mechanisms involving activation of the PHOSPHATIDYLINosIToL 3-KINASE/ protein Kinase akt pathway. ${ }^{43}$ Statins can also inhibit geranylgeranylation of Ras superfamily members, including Rho; however, activation of eNOS occurs much more rapidly via the phosphatidylinositol 3-kinase/protein kinase AKT signaling pathway, for which lower statin concentrations are required. ${ }^{44}$

The inhibitory effect of statins on Rac1 geranylgeranylation, which is involved in the production of reactive oxygen species by NADPH oxidase, is thought to maintain NO availability by preventing it from being degraded by free-radical molecules. Indeed, preclinical studies suggest that the effects of statin therapy in enhancing levels of NO might translate into important benefits for cerebral ischemia, by increasing blood flow ${ }^{45}$ and reducing neutrophil infiltration. ${ }^{46}$ In addition, the preservation of NO levels is implicated in additional effects of statin therapy on vascular cells, including promotion of angiogenesis and

\section{Box 1 Potential benefits and effects of statins in stroke}

\section{Improvement of endothelial dysfunction}

Increased synthesis of nitric oxide

Decreased synthesis of endothelin 1

Inhibition of LDL cholesterol oxidation

\section{Reduced inflammatory response}

Reduced number and activity of inflammatory cells

Reduced levels of C-reactive protein

Stabilization of atherosclerotic plaques

Reduced serum cholesterol levels

Reduced production of metalloproteinases 
Reduced thrombogenic response

Inhibition of platelet adhesion or aggregation

Reduced fibrinogen concentration

Reduced blood viscosity

attenuation of endothelial cell apoptosis. ${ }^{43}$ These effects might also lead to increased cerebral blood flow through increased collateral vessel formation.

\section{FUTURE APPROACHES WITH STATINS FOR STROKE PREVENTION AND TREATMENT}

Although statins reduce serum cholesterol levels and inflammation, the effects on each of these processes appear to be independent of one another ${ }^{47,48}$ This might have important clinical implications, as epidemiology studies, as well as clinical trials with statins, have shown that inflammation correlates better with stroke incidence than serum cholesterol levels. ${ }^{49}$ In the Pravastatin Inflammatory/CRP Evaluation (PRINCE) trial, ${ }^{49}$ pravastatin therapy resulted in reduced levels of C-reactive protein, which is a marker of inflammation. The reduced levels of C-reactive protein were observed soon after pravastatin initiation (within 12 weeks) and occurred independently of effects on LDL cholesterol levels. Preclinical studies of stroke in animal models have demonstrated that statin therapy can provide neuroprotective benefits, as well as improved neurorecovery. Simvastatin administered either before or 3-25 h after middle cerebral artery occlusion in a rodent model of stroke resulted in a dose-dependent decrease in the size of the infarction. ${ }^{45,50}$ Similarly, statins protected against ischemic stroke by decreasing the thrombogenic response in a thromboembolic model of stroke. ${ }^{51}$ In another laboratory, atorvastatin therapy initiated $24 \mathrm{~h}$ after middle cerebral artery occlusion in rats resulted in improved function and neurologic recovery, including evidence of angiogenesis, neurogenesis and synaptogenesis. ${ }^{52}$ Evidence of anti-inflammatory and neuroprotective effects of statins, as well as improved neuro-recovery, have led to the design of early phase-IB dose-escalation studies to evaluate the use of high-dose statins in acute stroke.

\section{EVIDENCE-BASED RECOMMENDATIONS FOR STROKE PREVENTION}

The American Heart Association and the National Stroke Association guidelines for the prevention of primary ischemic stroke focus on optimizing the management of modifiable risk factors, as well as identifying individuals with nonmodifiable risk factors. ${ }^{11,13}$ Both the American Heart Association and the National Stroke Association recommend using statins to prevent stroke for patients with elevated cholesterol concentrations and those with known CAD. The recommendations follow the National Cholesterol Education Program/Adult Treatment Panel III approach for managing elevated cholesterol levels, which includes lifestyle modifications. ${ }^{53}$

Although the lipid-modifying effects of statin therapy have been well documented, there is
increasing evidence that statins might have other beneficial effects, such as improving
endothelial function and anti-inflammatory effects, which could contribute to stroke risk
reduction. Stroke prevention guidelines have not yet been updated to reflect the results of some
of the newer clinical trials showing benefits for those at risk of stroke with normal cholesterol,
hypertension or diabetes. On the basis of these data, optimum therapies for stroke prevention
might include a statin in high-risk individuals, such as those with either hypertension or
diabetes, irrespective of their serum cholesterol levels. Furthermore, serum biomarkers, such
as C-reactive protein, which are independent of cholesterol levels, could potentially be used
in conjunction with standard risk assessments in order to provide a better evaluation of which 
individuals will benefit from statin therapy. Further studies will be needed, however, to determine the relative utility of C-reactive protein measurements over other traditional risk factors for stroke. Undoubtedly, future guidelines will have to reconsider these recommendations and consider making broader groups eligible for treatment with statins, on the basis of results from new trials.

\section{Acknowledgments}

RL Sacco is supported by grants from the National Institutes of Health. JK Liao is supported by grants from the National Institutes of Health and the American Heart Association Bugher Foundation.

\section{GLOSSARY}

G PROTEINS, GTP-binding regulatory proteins in the cell membrane that act as intermediaries between cell-surface receptors and intracellular enzymes, enabling regulation of enzyme activity in response to extracellular signals; RAS SUPERFAMILY, A superfamily of GTPbinding hydrolysis-coupled signal-transduction relay proteins that includes the Ras, Rho, Rab and Rap families; ISOPRENYLATION, Modification of intracellular signaling molecules by intermediates of the cholesterol biosynthesis pathway; PHOSPHATIDYLINOSITOL 3KINASE/PROTEIN KINASE AKT PATHWAY, Intracellular signaling pathway that mediates the effects of most growth factors; GERANYLGERANYLATION, An intermediate step of the cholesterol biosynthesis pathway, which is used to modify the Rho GTPase family of signaling molecules.

\section{References}

1. American Heart Association. Heart Disease and Stroke Statistics-2005 Update. 2005 [accessed 12 September 2005]. http://www.americanheart.org/presenter.jhtml?identifier=3000090

2. Liao JK, et al. Oxidized low-density lipoprotein decreases the expression of endothelial nitric oxide synthase. J Biol Chem 1995;270:319-324. [PubMed: 7529227]

3. Vergnani, et al. Effect of native and oxidized low-density lipoprotein on endothelial nitric oxide and superoxide production: key role of L-arginine availability. Circulation 2000;101:1261-1266. [PubMed: 10725285]

4. Iso H, et al. Serum cholesterol levels and six-year mortality from stroke in 350,977 men screened for the multiple risk factor intervention trial. N Engl J Med 1989;320:904-910. [PubMed: 2619783]

5. Kagan A, et al. Factors related to stroke incidence in Hawaii Japanese men. The Honolulu Heart Study. Stroke 1980;11:14-21. [PubMed: 6444469]

6. Leppala JM, et al. Different risk factors for different stroke subtypes: association of blood pressure, cholesterol, and antioxidants. Stroke 1999;30:2535-2540. [PubMed: 10582974]

7. Horenstein RB, et al. Cholesterol predicts stroke mortality in the Women's Pooling Project. Stroke 2002;33:1863-1868. [PubMed: 12105367]

8. Soyama Y, et al. High-density lipoprotein cholesterol and risk of stroke in Japanese men and women: the Oyabe Study. Stroke 2003;34:863-868. [PubMed: 12637692]

9. Sacco RL, et al. High-density lipoprotein cholesterol and ischemic stroke in the elderly: the Northern Manhattan Stroke Study. JAMA 2001;285:2729-2735. [PubMed: 11386928]

10. Tanne D, et al. Blood lipids and first-ever ischemic stroke/transient ischemic attack in the Bezafibrate Infarction Prevention (BIP) Registry: high triglycerides constitute an independent risk factor. Circulation 2001;104:2892-2897. [PubMed: 11739302]

11. Goldstein LB, et al. Primary prevention of ischemic stroke: a statement for healthcare professionals from the Stroke Council of the American Heart Association. Stroke 2001;32:280-299. [PubMed: 11136952]

12. Sacco RL. Risk factors, outcomes, and stroke subtypes for ischemic stroke. Neurology 1997;49 (Suppl):S39-S44. [PubMed: 9371148] 
13. Gorelick PB, et al. Prevention of a first stroke: a review of guidelines and a multidisciplinary consensus statement from the National Stroke Association. JAMA 1999;281:1112-1120. [PubMed: 10188663]

14. Landmesser U, et al. Simvastatin versus ezetimibe: pleiotropic and lipid-lowering effects on endothelial function in humans. Circulation 2005;111:2356-2363. [PubMed: 15867181]

15. Furberg CD, et al. Asymptomatic Carotid Artery Progression Study (ACAPS) Research Group. Effect of lovastatin on early carotid atherosclerosis and cardiovascular events. Circulation 1994;90:16791687. [PubMed: 7734010]

16. Crouse JR, et al. Pravastatin, Lipids, and Atherosclerosis in the Carotid Arteries (PLAC-II). Am J Cardiol 1995;75:455-459. [PubMed: 7863988]

17. MacMahon S, et al. LIPID Trial Research Group. Effects of lowering average of below-average cholesterol levels on the progression of carotid atherosclerosis: results of the LIPID Atherosclerosis Substudy. Circulation 1998;97:1784-1790. [PubMed: 9603532]

18. The Scandinavian Simvastatin Survival Study Investigators. Randomised trial of cholesterol lowering in 4444 patients with coronary heart disease: the Scandinavian Simvastatin Survival Study (4S). Lancet 1994;344:1383-1389. [PubMed: 7968073]

19. The Long-Term Intervention with Pravastatin in Ischemic Disease (LIPID) Study Group. Prevention of cardiovascular events and death with pravastatin in patients with coronary heart disease and a broad range of initial cholesterol levels. N Engl J Med 1998;339:1349-1357. [PubMed: 9841303]

20. Sacks FM, et al. Cholesterol and Recurrent Events Trial investigators. The effect of pravastatin on coronary events after myocardial infarction in patients with average cholesterol levels. N Engl J Med 1996;335:1001-1009. [PubMed: 8801446]

21. Heart Protection Study Collaborative Group. MRC/BHF Heart Protection Study of cholesterol lowering with simvastatin in 20,536 high-risk individuals: a randomised placebo-controlled trial. Lancet 2002;360:7-22. [PubMed: 12114036]

22. Collins R, et al. Effects of cholesterol-lowering with simvastatin on stroke and other major vascular events in 20536 people with cerebrovascular disease or other high-risk conditions. Lancet 2004;363:757-767. [PubMed: 15016485]

23. Shepherd J, et al. West of Scotland Coronary Prevention Study Group. Prevention of coronary heart disease with pravastatin in men with hypercholesterolemia. N Engl J Med 1995;333:1301-1307. [PubMed: 7566020]

24. Shepherd J, et al. Pravastatin in elderly individuals at risk of vascular disease (PROSPER): a randomised controlled trial. Lancet 2002;360:1623-1630. [PubMed: 12457784]

25. Sever PS, et al. Prevention of coronary and stroke events with atorvastatin in hypertensive patients who have average or lower-than-average cholesterol concentrations, in the Anglo-Scandinavian Cardiac Outcomes Trial-Lipid Lowering Arm (ASCOT-LLA): a multicentre randomised controlled trial. Lancet 2003;361:1149-1158. [PubMed: 12686036]

26. Colhoun HM, et al. Primary prevention of cardiovascular disease with atorvastatin in type 2 diabetes in the Collaborative Atorvastatin Diabetes Study (CARDS): multicentre randomised placebocontrolled trial. Lancet 2004;364:685-696. [PubMed: 15325833]

27. ALLHAT Officers and Coordinators for the ALLHAT Collaborative Research Group. Major outcomes in moderately hypercholesterolemic, hypertensive patients randomized to pravastatin vs usual care: the Antihypertensive and Lipid-Lowering Treatment to Prevent Heart Attack Trial (ALLHAT-LLT). JAMA 2002;288:2998-3007. [PubMed: 12479764]

28. Waters DD, et al. Effects of atorvastatin on stroke in patients with unstable angina or non-Q-wave myocardial infarction: a Myocardial Ischemia Reduction with Aggressive Cholesterol Lowering (MIRACL) substudy. Circulation 2002;106:1690-1695. [PubMed: 12270864]

29. Atkins D, et al. Cholesterol reduction and the risk for stroke in men. A meta-analysis of randomized, controlled trials. Ann Intern Med 1993;119:136-145. [PubMed: 8305001]

30. Blauw GJ, et al. Stroke, statins, and cholesterol. A meta-analysis of randomized, placebo-controlled, double-blind trials with HMG-CoA reductase inhibitors. Stroke 1997;28:946-950. [PubMed: 9158630]

31. Hebert PR, et al. Cholesterol lowering with statin drugs, risk of stroke, and total mortality. An overview of randomized trials. JAMA 1997;278:313-321. [PubMed: 9228438] 
32. Amarenco $P$, et al. Statins in stroke prevention and carotid atherosclerosis: systematic review and upto-date meta-analysis. Stroke 2004;35:2902-2909. [PubMed: 15514180]

33. Amarenco P, et al. Design and baseline characteristics of the stroke prevention by aggressive reduction in cholesterol levels (SPARCL) study. Cerebrovasc Dis 2003;16:389-395. [PubMed: 14584489]

34. Multiple Risk Factor Intervention Trial Research Group. Multiple risk factor intervention trial. Risk factor changes and mortality results. JAMA 1982;248:1465-1477. [PubMed: 7050440]

35. Sytkowski PA, et al. Changes in risk factors and the decline in mortality from cardiovascular disease. The Framingham Heart Study. N Engl J Med 1990;322:1635-1641. [PubMed: 2288563]

36. Prospective Studies Collaboration. Cholesterol, diastolic blood pressure, and stroke: 13,000 strokes in 450,000 people in 45 prospective cohorts. Lancet 1995;346:1647-1653. [PubMed: 8551820]

37. Tamai O, et al. Single LDL apheresis improves endothelium-dependent vasodilatation in hypercholesterolemic humans. Circulation 1997;95:76-82. [PubMed: 8994420]

38. Laufs U, et al. Suppression of endothelial nitric oxide production after withdrawal of statin treatment is mediated by negative feedback regulation of rho GTPase gene transcription. Circulation 2000;102:3104-3110. [PubMed: 11120702]

39. Cayatte AJ, et al. Chronic inhibition of nitric oxide production accelerates neointima formation and impairs endothelial function in hypercholesterolemic rabbits. Arterioscler Thromb 1994;14:753-759. [PubMed: 7513551]

40. Huang Z, et al. Enlarged infarcts in endothelial nitric oxide synthase knockout mice are attenuated by nitro-1-arginine. J Cereb Blood Flow Metab 1996;16:981-987. [PubMed: 8784243]

41. Laufs U, et al. Upregulation of endothelial nitric oxide synthase by HMG CoA reductase inhibitors. Circulation 1998;97:1129-1135. [PubMed: 9537338]

42. Laufs U, Liao JK. Post-transcriptional regulation of endothelial nitric oxide synthase mRNA stability by Rho GTPase. J Biol Chem 1998;273:24266-24271. [PubMed: 9727051]

43. Kureishi Y, et al. The HMG-CoA reductase inhibitor simvastatin activates the protein kinase Akt and promotes angiogenesis in normocholesterolemic animals. Nature Med 2000;6:1004-1010. [PubMed: 10973320]

44. Urbich C, et al. Double-edged role of statins in angiogenesis signaling. Circ Res 2002;90:737-744. [PubMed: 11934843]

45. Endres M, et al. Stroke protection by 3-hydroxy-3-methylglutaryl (HMG)-CoA reductase inhibitors mediated by endothelial nitric oxide synthase. Proc Natl Acad Sci USA 1998;95:8880-8885. [PubMed: 9671773]

46. Lefer AM, et al. Simvastatin preserves the ischemic-reperfused myocardium in normocholesterolemic rat hearts. Circulation 1999;100:178-184. [PubMed: 10402448]

47. Ridker PM, et al. Measurement of C-reactive protein for the targeting of statin therapy in the primary prevention of acute coronary events. N Engl J Med 2001;344:1959-1965. [PubMed: 11430324]

48. Ridker PM, et al. C-reactive protein levels and outcomes after statin therapy. N Engl J Med 2005;352:20-28. [PubMed: 15635109]

49. Albert MA, et al. Effect of statin therapy on C-reactive protein levels: the pravastatin inflammation/ CRP evaluation (PRINCE): a randomized trial and cohort study. JAMA 2001;286:64-70. [PubMed: 11434828]

50. Sironi L, et al. Treatment with statins after induction of focal ischemia in rats reduces the extent of brain damage. Arterioscler Thromb Vasc Biol 2003;23:322-327. [PubMed: 12588778]

51. Asahi M, et al. Protective effects of statins involving both eNOS and TPA in focal cerebral ischemia. J Cereb Blood Flow Metab 2005;25:722-729. [PubMed: 15716855]

52. Chen J, et al. Statins induce angiogenesis, neurogenesis, and synaptogenesis after stroke. Ann Neurol 2003;53:743-751. [PubMed: 12783420]

53. Expert Panel on Detection, Evaluation, And Treatment of High Blood Cholesterol In Adults. Executive Summary of The Third Report of The National Cholesterol Education Program (NCEP) Expert Panel on Detection, Evaluation, And Treatment of High Blood Cholesterol In Adults (Adult Treatment Panel III). JAMA 2001;285:2486-2497. [PubMed: 11368702] 
54. LaRosa JC, et al. Treating to New Targets (TNT) Investigators. Intensive lipid lowering with atorvastatin in patients with stable coronary disease. N Engl J Med 2005;352:1425-1435. [PubMed: 15755765]

55. Liao JK, Laufs U. Pleiotropic effects of statins. Annu Rev Pharmacol Toxicol 2005;45:89-118. [PubMed: 15822172] 
<smiles>CC(=O)SC(=O)CC(=O)SC#N</smiles>

\section{Acetyl-CoA + Acetoacetyl-CoA}<smiles>COC(=O)C[C@@](C)(O)CC(=O)SC=O</smiles><smiles>C[C@@](O)(CCO)CC(=O)O</smiles><smiles>CC(C)=CCC/C(C)=C/CC/C(C)=C/CP</smiles><smiles>CC(=O)O</smiles>

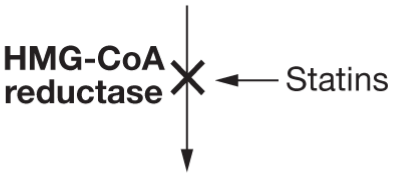

$\downarrow$ PI3 kinase/protein kinase AKT $\rightarrow \downarrow$ eNOS $\downarrow$ BMP2/bone formation $\downarrow$ Angiogenesis

Mevalonate



Figure 1.

Mechanisms underlying the pleiotropic effects of statins in stroke. A schematic of the cholesterol biosynthesis pathway showing the effects of inhibition of 3-hydroxy-3methylglutaryl-coenzyme A reductase by statins. Decreased isoprenylation of signaling molecules, such as Ras, Rho and Rac, leads to modulation of various signaling pathways. Reproduced with permission from reference 55 (C) (2005) Annual Reviews. BMP2, bone morphogenetic protein 2; Cdc42, cell division cycle 42 GTP-binding protein; CoA, coenzyme A; eNOS, endothelial nitric oxide synthase; ET1, endothelin-1; HMG, 3-hydroxy-3methylglutaryl; HO, hydroxy group; OPP, ether-linked pyrophosphate form of the isoprenoid; PAI1, plasminogen activator inhibitor 1; PI3 phosphatidylinositol 3; PP, pyrophosphate; tPA, tissue-type plasminogen activator. 


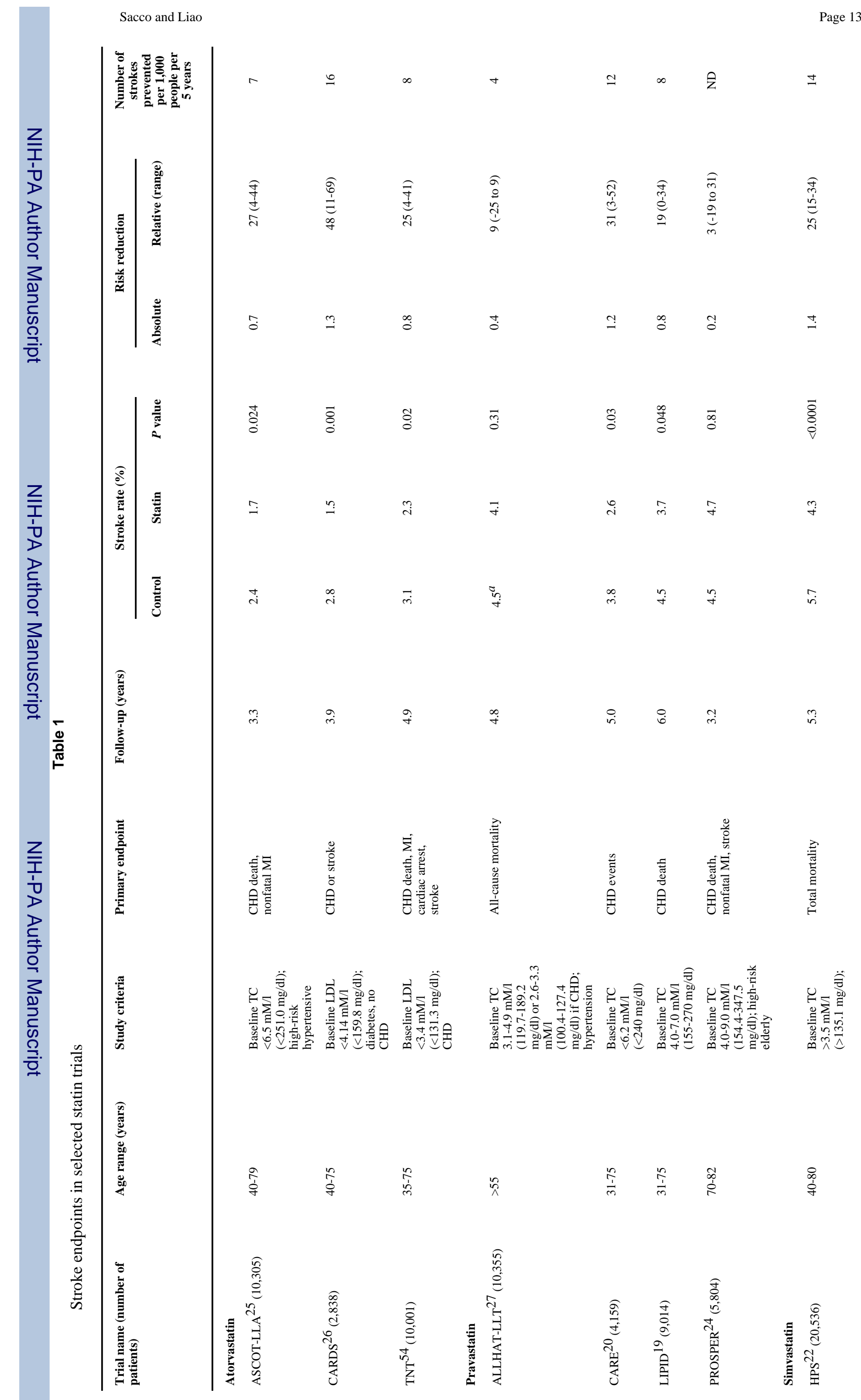




$$
1
$$

